\title{
Scattering-induced and highly tunable by gate damping-like spin-orbit torque in graphene doubly proximitized by two-dimensional magnet $\mathrm{Cr}_{2} \mathrm{Ge}_{2} \mathrm{Te}_{6}$ and monolayer $\mathrm{WS}_{2}$
}

\author{
Klaus Zollner $\odot,{ }^{1, *}$ Marko D. Petrović, ${ }^{2,3}$ Kapildeb Dolui $\odot,{ }^{3}$ Petr Plecháć, ${ }^{2}$ Branislav K. Nikolić $\odot,{ }^{3, \dagger}$ and Jaroslav Fabian $\odot^{1}$ \\ ${ }^{1}$ Institute for Theoretical Physics, University of Regensburg, 93040 Regensburg, Germany \\ ${ }^{2}$ Department of Mathematical Sciences, University of Delaware, Newark, Delaware 19716, USA \\ ${ }^{3}$ Department of Physics and Astronomy, University of Delaware, Newark, Delaware 19716, USA
}

(Received 14 May 2020; accepted 15 September 2020; published 9 October 2020)

\begin{abstract}
Graphene sandwiched between semiconducting monolayers of ferromagnet $\mathrm{Cr}_{2} \mathrm{Ge}_{2} \mathrm{Te}_{6}$ and transition-metal dichalcogenide $\mathrm{WS}_{2}$ acquires both spin-orbit (SO) coupling, of valley-Zeeman and Rashba types, and exchange coupling. Using first principles combined with quantum transport calculations, we predict that such doubly proximitized graphene within van der Waals heterostructure will exhibit SO torque driven by unpolarized charge current. This system lacks spin Hall current which is putatively considered as necessary for the efficient damping-like (DL) SO torque that plays a key role in magnetization switching. Instead, it demonstrates how a DL SO torque component can be generated solely by skew scattering off spin-independent potential barrier or impurities in purely two-dimensional electronic transport due to the presence of proximity SO coupling and its spin texture tilted out of plane. This leads to current-driven nonequilibrium spin density emerging in all spatial directions, whose cross product with proximity magnetization yields DL SO torque, unlike the ballistic regime with no scatterers in which only field-like (FL) SO torque appears. In contrast to SO torque on conventional metallic ferromagnets in contact with three-dimensional SO-coupled materials, the ratio of FL and DL components of SO torque can be tuned by more than an order of magnitude via combined top and back gates.
\end{abstract}

DOI: 10.1103/PhysRevResearch.2.043057

\section{INTRODUCTION}

The spin-orbit (SO) torque [1] is a phenomenon in which unpolarized charge current injected parallel to the interface of a bilayer of ferromagnetic metal (FM) and SO-coupled material induces magnetization dynamics of the FM layer. For many possible applications of torque [2], such as nonvolatile magnetic random access memories [3] or artificial neural networks [4], it is crucial to switch magnetization from up to down along the direction perpendicular to the interface. This has led to intense search for optimal SOcoupled materials and their interfaces with FM layers, which yield large SO torque while using as small as possible injected current. Thus far, minimal current density $j$ for magnetization switching has been achieved using topological insulators $\left(j \approx 6 \times 10^{5} \mathrm{~A} / \mathrm{cm}^{2}[5]\right)$ and Weyl semimetals ( $j \approx 3 \times 10^{5} \mathrm{~A} / \mathrm{cm}^{2}[6]$ ), which is two orders of magnitude smaller than $j$ required in early SO torque-operated devices employing heavy metals $[1,7]$.

Further optimization could be achieved by using van der Waals (vdW) heterostructures [8] of very recently dis-

\footnotetext{
*klaus.zollner@physik.uni-regensburg.de

†bnikolic@udel.edu

Published by the American Physical Society under the terms of the Creative Commons Attribution 4.0 International license. Further distribution of this work must maintain attribution to the author(s) and the published article's title, journal citation, and DOI.
}

covered two-dimensional (2D) ferromagnets [9,10] and 2D SO-coupled materials where current flows only through a few monolayers and no Joule heat is wasted by its flow through the bulk. Furthermore, vdW heterostructures offer atomically flat and highly transparent interfaces, as well as the possibility to use external manipulations-such as gating, straining, and controlling coupling between 2D materials - in order to tune [11] the magnitude and ratio of field-like (FL) and dampinglike (DL) components of SO torque. The traditional labeling of torque components stems from how they affect dynamics of magnetization $\mathbf{m}_{\mathrm{C}}$ viewed as a classical vector-FL torque changes precession around an effective magnetic field (such as along the $y$ axis in the case of vdW heterostructure in Fig. 1), while DL torque competes with damping and plays a key role [12] in magnetization switching (such by directing magnetization $\mathbf{m}_{\mathrm{C}}$ toward the $y$ axis in Fig. 1). Controlling their ratio and relative sign is of great interest for applications since it makes it possible to tailor the switching probability [13].

The critical problem in the field of SO torquesunderstanding of competing microscopic mechanisms behind these components, especially the DL one, and control of their magnitude and ratio-remains unresolved. For example, experiments on FM/heavy-metal bilayers suggest [7] that DL SO torque is primarily generated by the spin Hall current [14] from the bulk of a heavy metal, while interfacial SO coupling (SOC) is detrimental because it generates spin memory loss [15-17] and therefore reduction of spin Hall current. Conversely, first-principles quantum transport calculations $[18,19]$ on FM/heavy-metal bilayers find that interfacial SOC and 


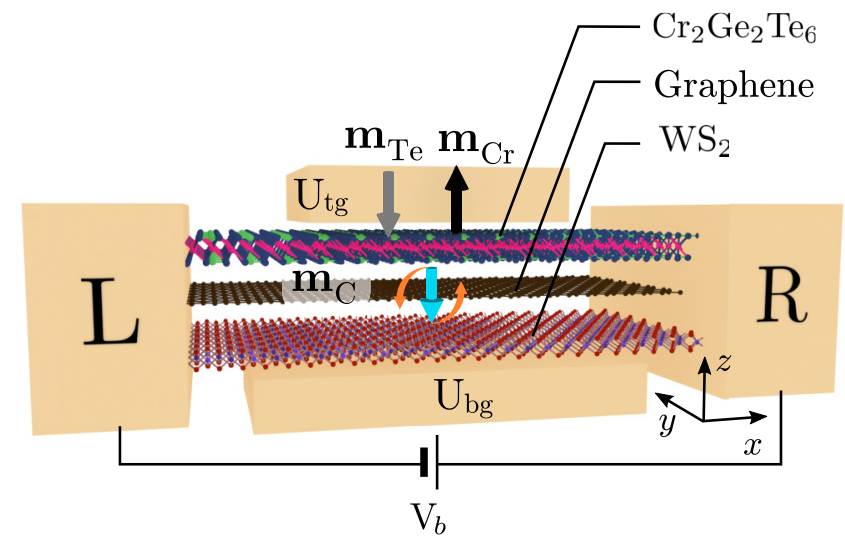

FIG. 1. Schematic view of $\mathrm{Cr}_{2} \mathrm{Ge}_{2} \mathrm{Te}_{6} /$ graphene/WS 2 vdW heterostructure attached to macroscopic left and right reservoirs with a small bias voltage $V_{b}$ between them injecting unpolarized charge current into graphene layer. A back-gate voltage $U_{\mathrm{bg}}$ and a top-gate voltage $U_{\text {tg }}$ applied over a smaller "active region" are assumed to control the Fermi energy and the local on-site potential in graphene, respectively. The unit vectors of magnetic moments on $\mathrm{Cr}$, Te, and $\mathrm{C}$ atoms are denoted as $\mathbf{m}_{\mathrm{Cr}}, \mathbf{m}_{\mathrm{Te}}$, and $\mathbf{m}_{\mathrm{C}}$, respectively, where only $\mathbf{m}_{\mathrm{C}}$ experiences SO torque-driven dynamics.

spin Hall current can equally contribute to total DL SO torque. The interfacial contribution is easily identified in experiments and computational studies as the one that is independent on the thickness of heavy-metal layer [18,19], but its microscopic mechanisms remain poorly understood. Although reflection and transmission of electron spins traversing SO-coupled interface can lead to interface-generated spin currents inducing DL SO torque [20,21], both this mechanism and the spin Hall current are inoperative in 2D transport within vdW heterostructures or in FM/topological-insulator bilayers [5,22]. Thus far, it has been argued that spin-independent impurities cannot generate DL torque in purely 2D transport [23-25], as well as that the same impurities can completely cancel [24,25] the intrinsic Berry curvature mechanism [26] of DL SO torque as the only other possibility previously discussed for purely 2D transport.

In this study, we design a realistic (i.e., built atom by atom) vdW heterostructure monolayer$\mathrm{Cr}_{2} \mathrm{Ge}_{2} \mathrm{Te}_{6} /$ graphene/monolayer-WS $\mathrm{WS}_{2}$ as depicted in Fig. 1. Here, $\mathrm{Cr}_{2} \mathrm{Ge}_{2} \mathrm{Te}_{6}[27,28]$ is an example of the recently discovered $2 \mathrm{D}$ ferromagnets $[9,10]$, and $\mathrm{WS}_{2}$ is a transition-metal dichalcogenide (TMD) with strong SOC in monolayer form [29]. Unlike isolated graphene, which is nonmagnetic and hosts minuscule intrinsic SOC [30], doubly proximitized graphene within a $\mathrm{Cr}_{2} \mathrm{Ge}_{2} \mathrm{Te}_{6} /$ graphene/ $\mathrm{WS}_{2}$ trilayer offers a versatile "theoretical laboratory" in which we can differentiate between competing mechanisms of SO torque and thereby learn how to control them. This can be accomplished by switching on and off different terms in the first-principles calculations (Figs. 2 and 3) derived from the Hamiltonian [Eq. (1)] of the heterostructure and then by performing quantum transport calculations.

The continuous version of such a Hamiltonian [Eq. (1)] can be used as an input for diagrammatic perturbative analytic calculations [24,25,31] based on the Kubo formula [32] for nonequilibrium spin density $\langle\hat{\mathbf{s}}(\mathbf{r})\rangle_{\mathrm{CD}}$ within graphene, which exerts torque $\propto\langle\hat{\mathbf{s}}(\mathbf{r})\rangle_{\mathrm{CD}} \times \mathbf{m}_{\mathrm{C}}(\mathbf{r})[8,18,33-35]$ on the local magnetization $\mathbf{m}_{\mathrm{C}}(\mathbf{r})$ in graphene. Note that $\mathbf{m}_{\mathrm{C}}(\mathbf{r})$ is induced by the magnetic proximity effect originating from $\mathrm{Te}$ atoms of $\mathrm{Cr}_{2} \mathrm{Ge}_{2} \mathrm{Te}_{6}$, so that $\mathbf{m}_{\mathrm{C}} \| \mathbf{m}_{\mathrm{Te}}$, but these two magnetic moments are antiparallel to $\mathbf{m}_{\mathrm{Cr}}$ on $\mathrm{Cr}$ atoms, as illustrated in Fig. 1.

Alternatively, one can discretize the continuous Hamiltonian in Eq. (1) to obtain its tight-binding (TB) version, as provided in Appendix D, and combine it with computational quantum transport. We feed such first-principles TB Hamiltonian into the calculations [33-35] of the current-driven (CD) part of nonequilibrium density matrix $\hat{\rho}_{\mathrm{CD}}$, which yields $\left\langle\hat{\mathbf{s}}_{i}\right\rangle_{\mathrm{CD}}=\operatorname{Tr}_{\text {spin }}\left[\hat{\rho}_{\mathrm{CD}} \hat{\mathbf{s}}\right]$ on site $i$ of TB lattice for $\hat{\mathbf{s}}=\left(\hat{s}_{x}, \hat{s}_{y}, \hat{s}_{z}\right)$ as the vector of the Pauli matrices and the trace being performed only in the spin space. The matrix $\hat{\rho}_{\mathrm{CD}}$ is computed via the nonequilibrium Green's function (NEGF) formalism [36] for the Landauer setup where the vdW heterostructure in Fig. 1 is divided into the left (L) semi-infinite lead connected to the "active region," which is connected to the right $(\mathrm{R})$ semi-infinite lead. Both semi-infinite leads and the active region in Fig. 1 are made of the same trilayer, assumed to be also infinite in the transverse $y$ direction. The leads terminate into the $\mathrm{L}$ and $\mathrm{R}$ macroscopic reservoirs kept at electrochemical potential difference $\mu_{\mathrm{L}}-\mu_{\mathrm{R}}=e V_{\mathrm{b}}$ with small bias voltage $V_{\mathrm{b}}$ in the linear-response regime. Such a nonperturbative (i.e., numerically exact) approach can be used to investigate $\mathrm{SO}$ torque in the diffusive transport regime $[18,35]$ as well as in the ballistic $[37,38]$ and quasiballistic regimes which are not accessible in the Kubo-formula-based calculations requiring nonzero electric field and momentum relaxation mechanisms. By using homogeneous potential barrier in the active region due to the top electrostatic gate $[39,40]$ in Fig. 1, and/or short-ranged impurities generating spin-independent random potential [31,41], we demonstrate (Fig. 4) our principal result - spin-independent scatterers can be a sole generator of nonzero DL SO torque in purely $2 \mathrm{D}$ transport, so they can be manipulated [31] to tune its magnitude.

The paper is organized as follows. Section II explains our density functional theory (DFT) calculations for $\mathrm{Cr}_{2} \mathrm{Ge}_{2} \mathrm{Te}_{6} /$ graphene/ $\mathrm{WS}_{2}$ trilayer, with additional details and cases (such as $\mathrm{Cr}_{2} \mathrm{X}_{2} \mathrm{Te}_{6} /$ graphene/ $\mathrm{WS}_{2}$ trilayers and $\mathrm{Cr}_{2} \mathrm{X}_{2} \mathrm{Te}_{6}$ /graphene bilayers where $\left.\mathrm{X}=\{\mathrm{Si}, \mathrm{Ge}, \mathrm{Sn}\}\right)$ provided in Appendixes A-C. Section II also provides a continuous effective Hamiltonian of graphene with parameters derived from DFT calculations, with its TB version given in Appendix D. The quantum transport calculations of currentdriven nonequilibrium spin densities and SO torques, based on the TB Hamiltonian (Appendix D) of doubly proximitized graphene with additional gate- or disorder-induced on-site potential, are presented in Sec. III. We conclude in Sec. IV.

\section{FIRST-PRINCIPLES BAND STRUCTURE AND THEREBY DERIVED LOW-ENERGY CONTINUOUS HAMILTONIAN}

Using DFT, we first compute the band structure (Figs. 2 and 3) of $\mathrm{Cr}_{2} \mathrm{Ge}_{2} \mathrm{Te}_{6} /$ graphene/ $/ \mathrm{WS}_{2}$ trilayer. Since the Dirac cones of graphene are largely preserved within the band gap of $\mathrm{Cr}_{2} \mathrm{Ge}_{2} \mathrm{Te}_{6}$, an effective Hamiltonian for graphene only can be 


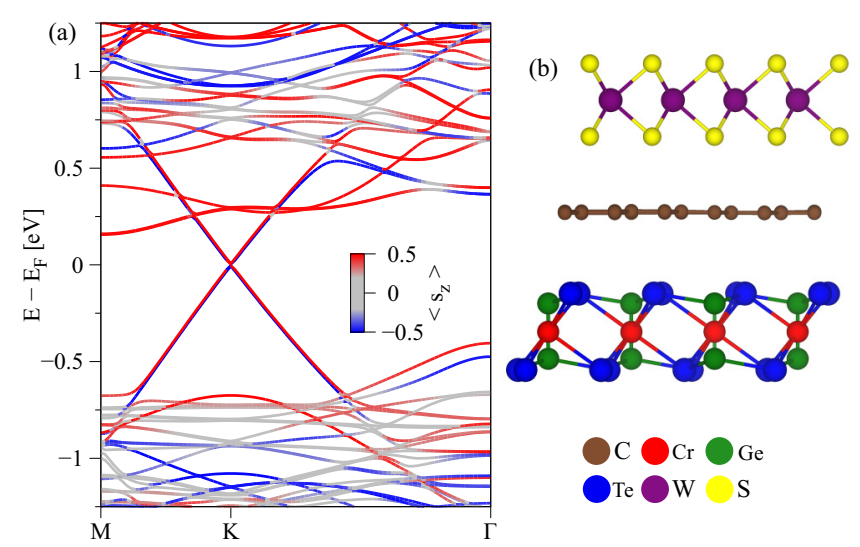

FIG. 2. (a) The DFT-calculated electronic band structure of graphene sandwiched between monolayer $\mathrm{Cr}_{2} \mathrm{Ge}_{2} \mathrm{Te}_{6}$ and monolayer $\mathrm{WS}_{2}$. The color corresponds to the $\left\langle\hat{s}_{z}\right\rangle$ expectation value in equilibrium. (b) Side view of the geometry of the trilayer supercell.

extracted in Eq. (1). The DFT calculations are performed on the supercell [Fig. 2(b)] of $\mathrm{Cr}_{2} \mathrm{Ge}_{2} \mathrm{Te}_{6} /$ graphene/WS 2 trilayer using QUANTUM ESPRESSO [42] and WIEN2K packages [43], with additional details provided in Appendix A. The band structure in Fig. 2(a) shows that the Dirac cone of graphene is preserved and located in the global band gap, so it can be probed by charge and spin transport. Figure 3 shows an enlargement of the fine structure around $\mathrm{K}$ and $\mathrm{K}^{\prime}$ points with a fit to our continuous Hamiltonian

$$
\begin{aligned}
\hat{H} & =\hat{H}_{0}+\hat{H}_{\Delta}+\hat{H}_{\mathrm{I}}+\hat{H}_{\mathrm{R}}+\hat{H}_{\mathrm{ex}}+\hat{H}_{\xi}, \\
\hat{H}_{0} & =\hbar v_{\mathrm{F}}\left(\tau k_{x} \hat{\sigma}_{x}-k_{y} \hat{\sigma}_{y}\right) \otimes \hat{s}_{0}, \\
\hat{H}_{\Delta} & =\Delta \hat{\sigma}_{z} \otimes \hat{s}_{0}, \\
\hat{H}_{\mathrm{I}} & =\tau\left(\lambda_{\mathrm{I}}^{\mathrm{A}} \hat{\sigma}_{+}+\lambda_{\mathrm{I}}^{\mathrm{B}} \hat{\sigma}_{-}\right) \otimes \hat{s}_{z}, \\
\hat{H}_{\mathrm{R}} & =-\lambda_{\mathrm{R}}\left(\tau \hat{\sigma}_{x} \otimes \hat{s}_{y}+\hat{\sigma}_{y} \otimes \hat{s}_{x}\right), \\
\hat{H}_{\mathrm{ex}} & =\left(-\lambda_{\mathrm{ex}}^{\mathrm{A}} \hat{\sigma}_{+}+\lambda_{\mathrm{ex}}^{\mathrm{B}} \hat{\sigma}_{-}\right) \otimes \hat{s}_{z}, \\
\hat{H}_{\xi} & =\tau \xi \hat{\sigma}_{0} \otimes \hat{s}_{0} .
\end{aligned}
$$

This is derived from first-principles calculations, as well as additional symmetry arguments [44,45], and it is valid in the vicinity of both Dirac points, $\mathrm{K}$ and $\mathrm{K}^{\prime}$. It reproduces the gap at the Dirac point and the spin splitting of the bands due to proximity SOC [46,47], of valley-Zeeman and Rashba types $[29,45,48]$, which originates from $\mathrm{WS}_{2}$, as well as the proximity magnetism $[49,50]$ from $\mathrm{Cr}_{2} \mathrm{Ge}_{2} \mathrm{Te}_{6}$ into graphene. These interactions are captured by relevant terms in our continuous Hamiltonian [Eq. (1)], with parameters provided in Table II.

The vdW heterostructure we consider has broken timereversal symmetry and only $C_{3}$ symmetry. We denote $v_{\mathrm{F}}$ as the Fermi velocity, and wave-vector components $k_{x}$ and $k_{y}$ are measured from $\mathrm{K}$ and $\mathrm{K}^{\prime}$. The valley index is $\tau= \pm 1$ for $\mathrm{K}$ and $\mathrm{K}^{\prime}$ and pseudospin matrices are $\hat{\sigma}_{i}$, acting on sublattice space $\left(\mathrm{C}_{\mathrm{A}}, \mathrm{C}_{\mathrm{B}}\right)$, with $i=\{0, x, y, z\}$, where $i=0$ denotes a unit $2 \times 2$ matrix. For notational convenience, we use $\hat{\sigma}_{ \pm}=$ $\frac{1}{2}\left(\hat{\sigma}_{z} \pm \hat{\sigma}_{0}\right)$. The staggered potential gap is $\Delta$, and the parameters $\lambda_{\mathrm{I}}^{\mathrm{A}}$ and $\lambda_{\mathrm{I}}^{\mathrm{B}}$ denote the sublattice-resolved intrinsic SOC. The parameter $\lambda_{\mathrm{R}}$ is the Rashba SOC, and the proximity exchange interaction parameters are $\lambda_{\mathrm{ex}}^{\mathrm{A}}$ and $\lambda_{\mathrm{ex}}^{\mathrm{B}}$. The param-
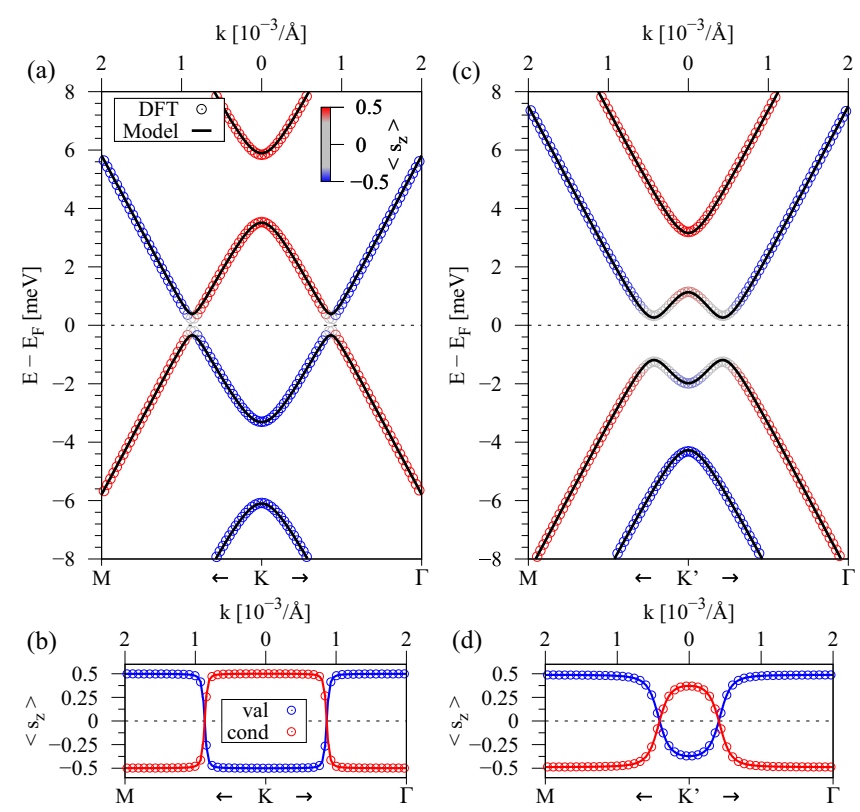

FIG. 3. (a) Enlargement of the low-energy bands around $E-E_{F}=0$ in Fig. 2. The Dirac cone is split by proximity-induced exchange interaction and SOC. Color of the bands corresponds to $\left\langle\hat{s}_{z}\right\rangle$ expectation value in equilibrium. (b) The $\left\langle\hat{s}_{z}\right\rangle$ expectation value of the valence (conduction) band in blue (red). Symbols are firstprinciples computed data and solid lines are fits using the continuous Hamiltonian in Eq. (1). [(c), (d)] The same information as in panels (a) and (b) but for $\mathrm{K}^{\prime}$ point.

eter $\xi$ describes valley exchange coupling resulting from an in-plane magnetization component [44]. The four basis states are $\left|\Psi_{\mathrm{A}}, \uparrow\right\rangle,\left|\Psi_{\mathrm{A}}, \downarrow\right\rangle,\left|\Psi_{\mathrm{B}}, \uparrow\right\rangle$, and $\left|\Psi_{\mathrm{B}}, \downarrow\right\rangle$. The continuous Hamiltonian is centered around the Fermi level at zero energy. Since first-principles results capture doping effects, we also introduce parameter $E_{\mathrm{D}}$ (termed Dirac point energy), which shifts the global band structure.

The fitting parameters for $\mathrm{Cr}_{2} \mathrm{Ge}_{2} \mathrm{Te}_{6} /$ graphene/WS trilayer are summarized in Table II. They are in agreement with previous calculations for individual graphene/TMD $[29,48]$ and graphene $/ \mathrm{Cr}_{2} \mathrm{Ge}_{2} \mathrm{Te}_{6}$ slabs [50,51]. As seen from Fig. 3, Hamiltonian in Eq. (1) with parameters from Table II perfectly reproduces first-principles results, including the spin $\left\langle\hat{s}_{z}\right\rangle$ expectation values in both valleys. The valley degeneracy is broken, which is best manifested at the highest (lowest) spin- $\uparrow\left(\right.$ spin- $\downarrow$ ) bands at $\mathrm{K}$ and $\mathrm{K}^{\prime}$. This is due to the interplay of the proximity exchange and SOC which splits the bands in the two valleys differently. Furthermore, the Rashba SOC mixes the spin states and opens a global band gap, different for the two valleys. Appendix $\mathrm{B}$ also provides equilibrium spin textures within $\mathrm{Cr}_{2} \mathrm{Ge}_{2} \mathrm{Te}_{6} /$ graphene/ $\mathrm{WS}_{2}$ trilayer. For comparison, band structure and fitting parameters for other $\mathrm{Cr}_{2} \mathrm{X}_{2} \mathrm{Te}_{6}$ /graphene/ $\mathrm{WS}_{2}$ trilayers and $\mathrm{Cr}_{2} \mathrm{X}_{2} \mathrm{Te}_{6}$ /graphene bilayers with $\mathrm{X}=\{\mathrm{Si}, \mathrm{Ge}, \mathrm{Sn}\}$ are provided in Appendix C.

\section{QUANTUM TRANSPORT CALCULATIONS OF SO TORQUE}

The NEGF is computed for the active region of $\mathrm{vdW}$ heterostructure in Fig. 1. Since current flows only through 

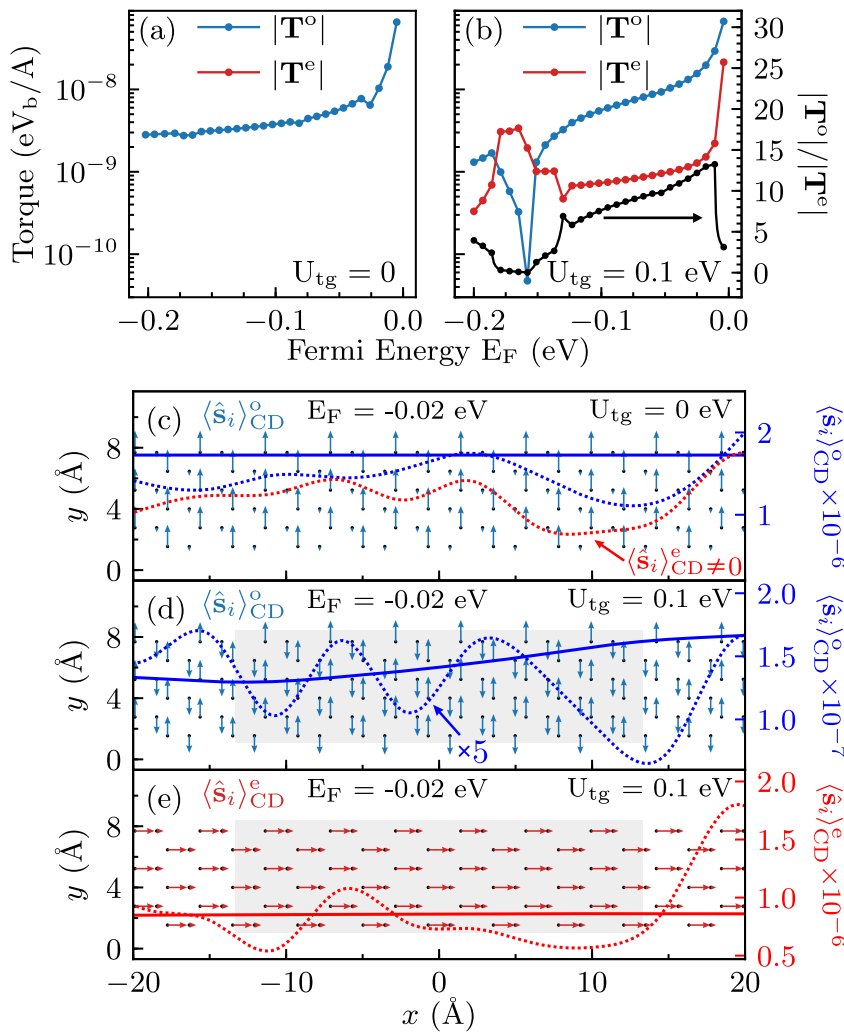

FIG. 4. The magnitude of odd $\left|\mathbf{T}^{\mathrm{o}}\right|$ and even $\left|\mathbf{T}^{\mathrm{e}}\right|$ SO torque components as a function of $E_{F}$ for proximity magnetization of graphene $\mathbf{m}_{\mathrm{C}} \| \hat{\mathbf{z}}$ (Fig. 1): (a) without the on-site energy $U_{\mathrm{tg}}=0$ and (b) with a constant on-site energy $U_{\mathrm{tg}}=0.1 \mathrm{eV}$ in the active region which creates a homogeneous potential barrier. The corresponding odd and even components of nonequilibrium spin density $\left\langle\hat{\mathbf{s}}_{i}\right\rangle_{\mathrm{CD}}^{\mathrm{e}, \mathrm{o}}$ [Eq. (2)] at $E_{F}=-0.02 \mathrm{eV}$ are shown as vector fields (c) without the barrier and [(d), (e)] with barrier $U_{\mathrm{tg}}=0.1 \mathrm{eV}$ within the gray rectangle. Solid lines in panels (c) and (d) show the magnitude of the sum of all arrows at coordinate $x$, while the dashed lines show how impurities modify the solid lines. The spin-independent impurities are modeled by random on-site energies $\in[-V / 2, V / 2]$ drawn from a uniform distribution with $V=3 \mathrm{eV}$ to ensure the diffusive transport regime (characterized by the shot noise Fano factor $F \simeq 1 / 3$ [56]). Each dashed line is geometric average over 20 disorder realizations.

graphene, the active region is modeled as graphene nanoribbon with armchair edges that is composed of $N_{a}=6$ dimer lines across the ribbon width $W$. The nanoribbon is described by the first-principles TB Hamiltonian [Eq. (D1)]. To remove the effect of the edges and mimic wide junctions along the $y$ axis often employed experimentally [39], sites on the lower and upper edges of the nanoribbon are connected [40] by a hopping with $e^{i k_{y} W}$ phase, where $k_{y} \in[-\pi / W, \pi / W)$. Thus, all transport quantities are then $k_{y}$ points sampled to take into account an infinitely wide system [40].

The conventional spin-transfer torque [33] in spin valves and magnetic tunnel junctions is decomposed into the FL and DL components, $\mathbf{T}=\mathbf{T}^{\mathrm{DL}}+\mathbf{T}^{\mathrm{FL}}$, each of which has relatively simple angular dependence. Conversely, the SO torque with its complex angular dependence [52] is naturally decomposed [18,35], $\mathbf{T}=\mathbf{T}^{\mathrm{e}}+\mathbf{T}^{\mathrm{o}}$, into the odd $\mathbf{T}^{\mathrm{o}}$ and even $\mathbf{T}^{\mathrm{e}}$ components in the magnetization $\mathbf{m}_{\mathrm{C}}$ receiving the torque.

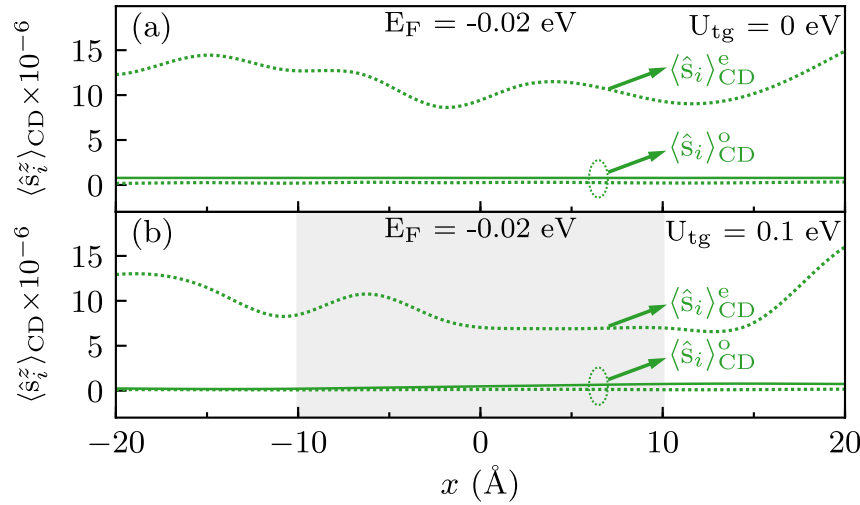

FIG. 5. Out-of-plane (i.e., along the $z$ axis in the coordinate system of Fig. 1) component of CD nonequilibrium spin density $\left\langle\hat{s}_{i}^{z}\right\rangle_{\mathrm{CD}}$ in doubly proximitized graphene with (a) no potential barrier $U_{\mathrm{tg}}=0 \mathrm{eV}$ or (b) with potential barrier due to homogeneous on-site energy $U_{\mathrm{tg}}=0.1 \mathrm{eV}$ within the region marked by the gray rectangle. Solid lines are for clean graphene, while dashed lines include spin-independent impurities modeled by random on-site energies $\in[-V / 2, V / 2]$ drawn from a uniform distribution with $V=3 \mathrm{eV}$ to ensure diffusive transport regime. Each dashed line is geometric average over 20 disorder realizations. The magnetization $\mathbf{m}_{\mathrm{C}}$ is oriented along the $z$ axis and the Fermi energy is $E_{F}=-0.02 \mathrm{eV}$. The corresponding in-plane components of $\left\langle\hat{\mathbf{s}}_{i}\right\rangle_{\mathrm{CD}}$ are shown in Figs. 4(c)-4(e). The arrows denote how different $\left\langle\hat{s}_{i}^{z}\right\rangle_{\mathrm{CD}}$ curves contribute to $\left\langle\hat{\mathbf{s}}_{i}\right\rangle_{\mathrm{CD}}^{\mathrm{e}}$ or $\left\langle\hat{\mathbf{s}}_{i}\right\rangle_{\mathrm{CD}}^{\mathrm{o}}$ vectors.

They are obtained by averaging over $N_{\mathrm{A}}$ atoms of the triangular sublattice A and $N_{\mathrm{B}}$ atoms of the triangular sublattice B of graphene

$$
\begin{aligned}
\mathbf{T}^{\mathrm{e}, \mathrm{o}}\left(k_{y}\right)= & \frac{1}{N_{\mathrm{A}}} \sum_{i \in \mathrm{A}}\left(-\frac{2 \lambda_{\mathrm{ex}}^{\mathrm{A}}}{\hbar}\right)\left\langle\hat{\mathbf{s}}_{i}\right\rangle_{\mathrm{CD}}^{\mathrm{e}, \mathrm{o}}\left(k_{y}\right) \times \mathbf{m}_{\mathrm{C}} \\
& +\frac{1}{N_{\mathrm{B}}} \sum_{j \in \mathrm{B}} \frac{2 \lambda_{\mathrm{ex}}^{\mathrm{B}}}{\hbar}\left\langle\hat{\mathbf{s}}_{j}\right\rangle_{\mathrm{CD}}^{\mathrm{e}, \mathrm{o}}\left(k_{y}\right) \times \mathbf{m}_{\mathrm{C}} .
\end{aligned}
$$

The total torque is the sum over the first Brillouin zone (BZ), $\mathbf{T}^{\mathrm{e}, \mathrm{o}}=\frac{W}{2 \pi} \int_{\mathrm{BZ}} \mathbf{T}^{\mathrm{e}, \mathrm{o}}\left(k_{y}\right) d k_{y}$. The NEGF-based algorithm to split the density matrix, $\hat{\rho}_{\mathrm{CD}}=\hat{\rho}_{\mathrm{CD}}^{\mathrm{e}}+\hat{\rho}_{\mathrm{CD}}^{\mathrm{o}}$, is given in Refs. [33,34], so that tracing Pauli matrices with $\hat{\rho}_{\mathrm{CD}}^{\mathrm{e}}$ and $\hat{\rho}_{\mathrm{CD}}^{\mathrm{o}}$ yields directly $\left\langle\hat{\mathbf{s}}_{i}\right\rangle_{\mathrm{CD}}^{\mathrm{e}}$ and $\left\langle\hat{\mathbf{s}}_{i}\right\rangle_{\mathrm{CD}}^{\mathrm{o}}$ and the corresponding SO torque components in Eq. (2).

The magnitudes of odd and even SO torque components are plotted in Figs. 4(a) and 4(b) in the units of $\mathrm{eV}_{b} / A$, where $A$ is the area of a single hexagon of the honeycomb lattice. We assume that back gate and top gate can change the carrier density globally or locally, respectively, thereby making it possible to tune the Fermi energy $E_{F}$ of the whole device while concurrently creating a potential barrier within its smaller region, as demonstrated experimentally [39]. Without a potential barrier, $U_{\mathrm{tg}}=0$, and in the ballistic regime with no impurities, the only nonzero component in Fig. 4(a) is $\mathbf{T}^{\mathbf{0}} \neq 0$. Upon introducing $U_{\mathrm{tg}} \neq 0$ in still clean graphene, Fig. 4(b) reveals emergence of $\mathbf{T}^{\mathrm{e}} \neq 0$. Furthermore, combined tuning of $E_{F}$ and potential barrier height in Fig. 4(b) modulates the ratio $\left|\mathbf{T}^{\mathrm{o}}\right| /\left|\mathbf{T}^{\mathrm{e}}\right|$ by an order of magnitude, which is of great importance for applications [13]. We fix the height of the 
homogeneous barrier, within the gray rectangle of the active region region in Figs. 4(d) and 4(e), at $U_{\mathrm{tg}}=0.1 \mathrm{eV}$.

To clarify how $\mathbf{T}^{\mathrm{e}}$ emerges from zero value in graphene without any scattering to nonzero value, Fig. 4(c) and Figs. 4(d) and 4(e) plot spatial profiles of nonequilibrium spin densities $\left\langle\hat{\mathbf{s}}_{i}\right\rangle_{\mathrm{CD}}^{\mathrm{e}, \mathrm{o}}$ [Eq. (2)] in the absence or presence of potential barrier, respectively. In Fig. 4(c), only $\left\langle\hat{\mathbf{s}}_{i}\right\rangle_{\mathrm{CD}}^{\circ} \neq 0$ is nonzero along the $y$ axis and slightly out of the plane in Fig. 5(a) for current injected along the $x$ axis. This is the wellknown inverse spin-galvanic (or Edelstein) effect [53-55] in which current through 2D electron system with SOC, and thereby generated spin texture with spin-momentum locking, induces $\left\langle\hat{\mathbf{s}}_{i}\right\rangle_{\mathrm{CD}} \neq 0$. But here this effect is operative in the ballistic transport regime $[37,38]$, instead of originally considered diffusive regime [53,54]. Upon introducing impurities [leading to dashed red line in Fig. 4(c)], as random on-site potential to establish the diffusive transport regime characterized by the shot noise Fano factor $F \simeq 1 / 3$ [56], $\left\langle\hat{\mathbf{s}}_{i}\right\rangle_{\mathrm{CD}}$ acquires additional components in the other two spatial directions. The same occurs in backscattering from a barrier [leading to solid red line in Fig. 4(e)]. This means that components of $\left\langle\hat{\mathbf{s}}_{i}\right\rangle_{\mathrm{CD}}$ emerge along the $x$ axis [Figs. 4(c) and 4(e)] and the $z$ axis (Fig. 5) to constitute $\left\langle\hat{\mathbf{s}}_{i}\right\rangle_{\mathrm{CD}}^{\mathrm{e}}$ vector. Thus, scattering off either the barrier or impurities is required to produce $\left\langle\hat{\mathbf{s}}_{i}\right\rangle_{\mathrm{CD}}^{\mathrm{e}}$ and $\mathbf{T}^{\mathrm{e}} \neq 0$.

Previously discussed mechanisms [23-25] for purely interfacial $\mathbf{T}^{\mathrm{e}} \neq 0$ require spin-active impurities, such as magnetic or SO ones [57], that scatter spin- $\uparrow$ and spin- $\downarrow$ electrons differently. However, the potential barrier and/or short-ranged impurities we employ in Fig. 4 are spin independent. Nonetheless, they can generate skew scattering $[31,41]$ due to proximity SOC in the band structure and sublattice-staggered potentials in Eq. (1) which conspire to tilt (Fig. 7) the equilibrium spin textures out of the plane. This occurs even when $\mathbf{m}_{C}=0$, while the barrier also requires $\mathbf{m}_{\mathrm{C}} \neq 0$. Both cases lead to generation of $\left\langle\hat{\mathbf{s}}_{\boldsymbol{i}}\right\rangle_{\mathrm{CD}}$ components in all spatial directions.

This is further clarified by Figs. 5(a) and 5(b) complementing Fig. 4(c) and Figs. 4(d) and 4(e), respectively-which reveal the out-of-plane component of CD nonequilibrium spin density $\left\langle\hat{s}_{i}^{z}\right\rangle_{\mathrm{CD}}$. Thus, spin-dependent skew scattering [41] generates a large out-of-plane component $\left\langle\hat{s}_{i}^{z}\right\rangle_{\mathrm{CD}}$ (dashed lines) in Fig. 5 which contributes to $\left\langle\hat{\mathbf{s}}_{i}\right\rangle_{\mathrm{CD}}^{\mathrm{e}}$ and the corresponding even component of SO torque, $\mathbf{T}^{\mathrm{e}}$, via Eq. (2).

To connect $\mathbf{T}^{\mathrm{e}}$ and $\mathbf{T}^{\mathrm{o}}$ to traditionally discussed DL and FL torque components, we need to take into account typically complex angular dependence of SO torque observed experimentally [52] or in first-principles quantum transport studies $[8,18,35]$. For this purpose, we fit computational data (dots in Fig. 6) with an infinite series $[8,35,52]$ for $\mathbf{T}^{\mathrm{e}}$ and $\mathbf{T}^{\mathrm{o}}$ vector fields on the unit sphere of orientations of $\mathbf{m}_{\mathrm{C}}$. The non-negligible terms, compatible with the lattice symmetry, are given by

$$
\begin{aligned}
\mathbf{T}^{\mathrm{o}} & =\left[\tau_{0}^{\mathrm{o}}+\tau_{2}^{\mathrm{o}}\left|\hat{\mathbf{z}} \times \mathbf{m}_{\mathrm{C}}\right|^{2}+\tau_{4}^{\mathrm{o}}\left|\hat{\mathbf{z}} \times \mathbf{m}_{\mathrm{C}}\right|^{4}\right]\left(\hat{\mathbf{y}} \times \mathbf{m}_{\mathrm{C}}\right) \\
\mathbf{T}^{\mathrm{e}} & =\left[\tau_{0}^{\mathrm{e}}+\tau_{2}^{\mathrm{e}}\left|\hat{\mathbf{z}} \times \mathbf{m}_{\mathrm{C}}\right|^{2}+\tau_{4}^{\mathrm{e}}\left|\hat{\mathbf{z}} \times \mathbf{m}_{\mathrm{C}}\right|^{4}\right] \mathbf{m}_{\mathrm{C}} \times\left(\hat{\mathbf{y}} \times \mathbf{m}_{\mathrm{C}}\right)
\end{aligned}
$$

The computed angular dependence of even, $\mathbf{T}^{\mathrm{e}}$, and odd, $\mathbf{T}^{\mathrm{o}}$, SO torque components-as the magnetization vector $\mathbf{m}_{\mathrm{C}}=(\sin \theta \cos \varphi, \sin \theta \sin \varphi, \cos \theta)$ rotates within the $x z, x y$,
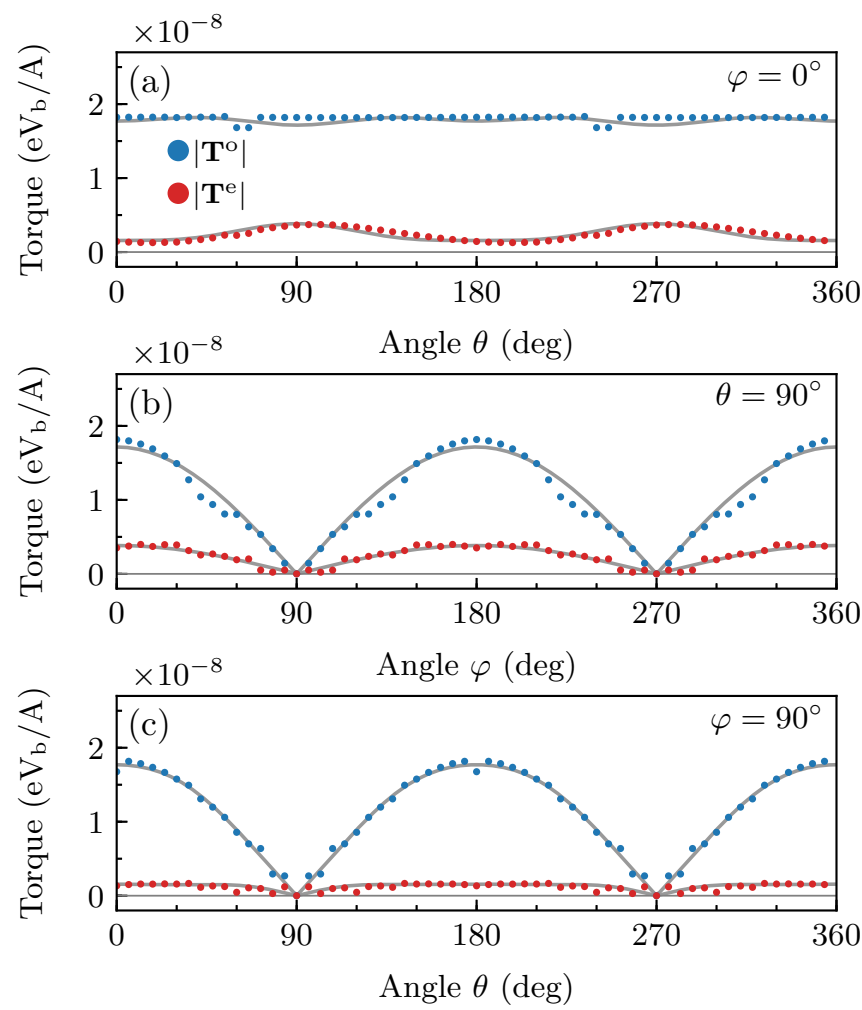

FIG. 6. [(a)-(c)] The angular dependence of SO torque components $\left|\mathbf{T}^{\mathrm{o}}\right|$ (blue dots) and $\left|\mathbf{T}^{\mathrm{e}}\right|$ (red dots) for different orientations of magnetization $\mathbf{m}_{\mathrm{C}}$ of proximitized clean graphene at $E_{F}=-0.02 \mathrm{eV}$ and with potential barrier in the active region due to on-site potential $U_{\text {tg }}=0.1 \mathrm{eV}$. Gray lines in the background fit, using Eq. (3), numerically calculated $\mathrm{SO}$ torque values (dots). The vector $\mathbf{m}_{\mathrm{C}}=$ ( $\sin \theta \cos \varphi, \sin \theta \sin \varphi, \cos \theta$ ) rotates within the $x z$ plane in panel (a); within the $x y$ plane in panel (b); and within the $y z$ plane in panel (c).

and $y z$ planes in the setup of Fig. 1 with nonzero potential barrier-is shown by dots in Fig. 6 . Note that for $\theta=0^{\circ}$, the results in Fig. 6 correspond to the results in Fig. 4(b) at $E_{F}=$ $-0.02 \mathrm{eV}$. The functions in Eq. (3), with fitting parameters listed in Table I, are then plotted as solid lines in Fig. 6. Here $\hat{\mathbf{y}}$ and $\hat{\mathbf{z}}$ are the unit vectors along the $y$ and $z$ axes, respectively, for the coordinate system in Fig. 1. Note that prior to fitting, data sets in all three planes $(x z, x y$, and $y z)$ are joined in a single continuous line for each case (with and without the on-site potential $U_{\mathrm{tg}}$ ), so that they could all be fitted with a single set of fitting parameters.

In Eq. (3), we recognize the lowest-order term $\tau_{0}^{0} \hat{\mathbf{y}} \times \mathbf{m}_{\mathrm{C}}$ as the FL SO torque, which is the only term (Table I) generated in the ballistic transport regime of Figs. 4(a) and 4(c). The usual DL SO torque [1] is $\tau_{0}^{\mathrm{e}} \mathbf{m}_{\mathrm{C}} \times\left(\hat{\mathbf{y}} \times \mathbf{m}_{\mathrm{C}}\right)$. Higher order terms in Eq. (3) can have properties of both FL and DL torques [18]. Fitted functions in Eq. (3) are also required for using [8] computed SO torque in micromagnetic simulations [12] of the dynamics of $\mathbf{m}_{\mathrm{C}}(t)$.

\section{CONCLUSIONS}

In conclusion, using first-principles calculations we design a vdW heterostructure where graphene is sandwiched between semiconducting monolayers of ferromagnet $\mathrm{Cr}_{2} \mathrm{Ge}_{2} \mathrm{Te}_{6}$ 
TABLE I. The fitting parameters in Eq. (3).

\begin{tabular}{|c|c|c|c|c|c|c|}
\hline $\begin{array}{l}U_{\mathrm{tg}} \\
(\mathrm{eV})\end{array}$ & $\begin{array}{c}\tau_{0}^{\mathrm{o}} \\
\left(\mathrm{eV}_{\mathrm{b}} / \mathrm{A}\right)\end{array}$ & $\begin{array}{c}\tau_{2}^{\mathrm{o}} \\
\left(\mathrm{eV}_{\mathrm{b}} / \mathrm{A}\right)\end{array}$ & $\begin{array}{c}\tau_{4}^{\mathrm{o}} \\
\left(\mathrm{eV}_{\mathrm{b}} / \mathrm{A}\right)\end{array}$ & $\begin{array}{c}\tau_{0}^{\mathrm{e}} \\
\left(\mathrm{eV}_{\mathrm{b}} / \mathrm{A}\right)\end{array}$ & $\begin{array}{c}\tau_{2}^{\mathrm{e}} \\
\left(\mathrm{eV}_{\mathrm{b}} / \mathrm{A}\right)\end{array}$ & $\begin{array}{c}\tau_{4}^{\mathrm{e}} \\
\left(\mathrm{eV}_{\mathrm{b}} / \mathrm{A}\right)\end{array}$ \\
\hline 0.0 & $1.112 \times 10^{-8}$ & 0.0 & 0.0 & 0.0 & 0.0 & 0.0 \\
\hline 0.1 & $1.770 \times 10^{-8}$ & $2.416 \times 10^{-9}$ & $-2.967 \times 10^{-9}$ & $1.560 \times 10^{-9}$ & $1.234 \times 10^{-11}$ & $2.262 \times 10^{-9}$ \\
\hline
\end{tabular}

and TMD $\mathrm{WS}_{2}$ to acquire both SOC, of valley-Zeeman and Rashba types, and exchange couplings via the respective proximity effects. Such doubly proximitized graphene offers a playground to investigate different microscopic mechanisms of SO torque. We provide first-principles continuous and tight-binding effective Hamiltonians of doubly proximitized graphene which can be used as a starting point of analytical diagrammatic or computational quantum transport calculations, respectively. In particular, using computational quantum transport, we demonstrate how the DL component of SO torque can be generated solely by skew scattering off spin-independent potential barrier or impurities in purely two-dimensional electronic transport due to the presence of proximity SOC and its spin texture tilted out of plane. This occurs without any mechanisms based on the spin Hall effect [7] or three-dimensional transport across the interface [20,21], which are naturally absent from the vdW heterostructure.

\section{ACKNOWLEDGMENTS}

K.Z. and J.F. were supported by the Deutsche Forschungsgemeinschaft (DFG, German Research Foundation) SFB 1277 (Project Id. No. 314695032) and SPP 1666. M.D.P. and P.P. were supported by ARO MURI Award No. W911NF14-0247. K.D. and B.K.N. were supported by the U.S. Department of Energy (DOE) Grant No. DE-SC0016380. The supercomputing time was provided by XSEDE, which is supported by the U.S. National Science Foundation (NSF) Grant No. ACI-1053575.

\section{APPENDIX A: COMPUTATIONAL DETAILS OF DFT CALCULATIONS}

The electronic structure calculations and structural relaxation for graphene on $\mathrm{Cr}_{2} \mathrm{X}_{2} \mathrm{Te}_{6}$, as well as for graphene sandwiched between $\mathrm{WS}_{2}$ and $\mathrm{Cr}_{2} \mathrm{X}_{2} \mathrm{Te}_{6}$ with $\mathrm{X}=\{\mathrm{Si}, \mathrm{Ge}, \mathrm{Te}\}$, are performed by DFT implemented in QUANTUM ESPRESSO package [42]. The heterostructure supercell in Fig. 2(b) consists of $5 \times 5$ supercell of graphene whose bottom surface is covered by a $\sqrt{3} \times \sqrt{3}$ supercell of $\mathrm{Cr}_{2} \mathrm{Ge}_{2} \mathrm{Te}_{6}$, while its top surface is covered by a $4 \times 4$ supercell of $\mathrm{WS}_{2}$. We stretch the lattice constant of graphene by roughly $2 \%$-from 2.46 to $2.5 \AA$ - and stretch the lattice constant of $\mathrm{Cr}_{2} \mathrm{Ge}_{2} \mathrm{Te}_{6}$ by roughly $6 \%$-from $6.8275 \AA$ [28] to $7.2169 \AA$. The $\mathrm{WS}_{2}$ lattice constant is compressed by roughly $1 \%$-from $3.153 \AA$ [58] to $3.125 \AA$. The heterostructure supercell has a lattice constant of $12.5 \AA$ and it contains 128 atoms. The distance between $\mathrm{WS}_{2}$ and graphene is $\simeq 3.28 \AA$, in agreement with previous calculations [29]. The distance between $\mathrm{Cr}_{2} \mathrm{Ge}_{2} \mathrm{Te}_{6}$ and graphene is about $3.52 \AA$, also in agreement with previous calculations [51].
Bulk vdW crystal $\mathrm{Cr}_{2} \mathrm{Ge}_{2} \mathrm{Te}_{6}$, which is composed of weakly bound monolayers, has Curie temperature $T_{\mathrm{C}} \simeq 60 \mathrm{~K}$ and PMA. Each monolayer is formed by edgesharing $\mathrm{CrTe}_{6}$ octahedra where Ge pairs are located in the hollow sites formed by the octahedra honeycomb. The layers are $\mathrm{ABC}$ stacked, resulting in a rhombohedral $R \overline{3}$ symmetry.

For bilayer (trilayer) structures we use $24 \times 24 \times 1(18 \times$ $18 \times 1) k$-point grid in self-consistent calculations to ensure converged results for the proximity exchange interaction and proximity SOC. We also perform open-shell calculations that provide the spin-polarized ground state of $\mathrm{Cr}_{2} \mathrm{Ge}_{2} \mathrm{Te}_{6}$. The Hubbard $U=1 \mathrm{eV}$ is used for $\mathrm{Cr} d$ orbitals, being in the range of proposed $U$ values for this compound [27]. The Perdew-Burke-Ernzerhof parametrization [59] of the generalized gradient approximation for the exchange-correlation functional is employed. We use an energy cutoff for charge density of $500 \mathrm{Ry}$, and the kinetic energy cutoff for wave functions is $60 \mathrm{Ry}$ for the scalar relativistic pseudopotential within the projector augmented wave method [60] describing electron-core interactions. When SOC is included, we use the relativistic versions of the pseudopotentials.

For the relaxation of the heterostructures, we add vdW corrections [61,62] and use quasi-Newton algorithm based on trust radius procedure. In order to simulate quasi-twodimensional systems, we add a vacuum of $20 \AA$, which avoids interactions between periodic images in the slab geometry. To determine the interlayer distances, the atoms of graphene and $\mathrm{WS}_{2}$ are allowed to relax only along the $z$ axis (perpendicular to the layers), while the atoms of $\mathrm{Cr}_{2} \mathrm{Ge}_{2} \mathrm{Te}_{6}$ are allowed to move in all directions, until all components of all forces are reduced below $10^{-3} \mathrm{Ry} / a_{0}$ ( $a_{0}$ is the Bohr radius).

Table II summarizes fitting parameters in the continuous Hamiltonian in Eq. (1) for graphene within $\mathrm{Cr}_{2} \mathrm{X}_{2} \mathrm{Te}_{6} /$ graphene/WS 2 trilayers with $\mathrm{X}=\{\mathrm{Si}, \mathrm{Ge}, \mathrm{Sn}\}$. The same parameters are also used in the TB version [Eq. (D1)] of the continuous Hamiltonian.

\section{APPENDIX B: EQUILIBRIUM SPIN TEXTURES IN $\mathrm{Cr}_{2} \mathrm{Ge}_{2} \mathrm{Te}_{6} /$ GRAPHENE/WS 2 TRILAYER}

Figure 7 shows DFT-calculated equilibrium spin textures (i.e., expectation values of the Pauli matrices in the eigenstates of the DFT Hamiltonian) for $\mathrm{Cr}_{2} \mathrm{Ge}_{2} \mathrm{Te}_{6}$ /graphene/WS $\mathrm{W}_{2}$ trilayer, with proximity magnetization of graphene pointing out of the plane [Figs. 7(a)-7(d)] or in the plane [Figs. 7(e)-7(h)]. The textures are plotted in the $k_{x} k_{y}$ plane in the vicinity of the $\mathrm{K}$ and $\mathrm{K}^{\prime}$ points at energies belonging to the conduction and valence bands, respectively. Thus, they complement the low-energy band structure shown in Fig. 3. The bands are strongly $s_{z}$ polarized with some small in-plane contribution. 
TABLE II. The fitting parameters of continuous Hamiltonian in Eq. (1) for $\mathrm{Cr}_{2} \mathrm{X}_{2} \mathrm{Te}_{6} /$ graphene/WS $\mathrm{W}_{2}$ trilayers with $\mathrm{X}=\{\mathrm{Si}, \mathrm{Ge}, \mathrm{Te}\}$. Here $v_{\mathrm{F}}$ is the Fermi velocity; $\Delta$ is the staggered potential gap; $\lambda_{\mathrm{R}}$ is the Rashba SOC strength; $\lambda_{\mathrm{I}}^{\mathrm{A}}$ and $\lambda_{\mathrm{I}}^{\mathrm{B}}$ are sublattice-resolved intrinsic SOC parameters; $\lambda_{\mathrm{ex}}^{\mathrm{A}}$ and $\lambda_{\mathrm{ex}}^{\mathrm{B}}$ are sublattice-resolved exchange interaction parameters; $\xi$ is the valley exchange parameter; and $E_{\mathrm{D}}$ is the Dirac point energy. The values of parameters fit the DFT-computed bands both with and without SOC.

\begin{tabular}{|c|c|c|c|c|c|c|c|c|c|c|}
\hline Calc. & $\mathrm{X}$ & $\begin{array}{c}v_{\mathrm{F}} \\
{\left[10^{5} \frac{\mathrm{m}}{\mathrm{s}}\right]}\end{array}$ & $\begin{array}{c}\Delta \\
{[\mathrm{meV}]}\end{array}$ & $\begin{array}{c}\lambda_{\mathrm{ex}}^{\mathrm{A}} \\
{[\mathrm{meV}]}\end{array}$ & $\begin{array}{c}\lambda_{\mathrm{ex}}^{\mathrm{B}} \\
{[\mathrm{meV}]}\end{array}$ & $\begin{array}{c}\lambda_{\mathrm{R}} \\
{[\mathrm{meV}]}\end{array}$ & $\begin{array}{c}\lambda_{\mathrm{I}}^{\mathrm{A}} \\
{[\mathrm{meV}]}\end{array}$ & $\begin{array}{c}\lambda_{\mathrm{I}}^{\mathrm{B}} \\
{[\mathrm{meV}]}\end{array}$ & $\begin{array}{c}\xi \\
{[\mathrm{meV}]}\end{array}$ & $\begin{array}{c}E_{\mathrm{D}} \\
{[\mathrm{meV}]}\end{array}$ \\
\hline No SOC & $\mathrm{Si}$ & 7.921 & 0.865 & -1.899 & -1.811 & & & & & -0.012 \\
\hline No SOC & $\mathrm{Ge}$ & 7.901 & 1.326 & -3.644 & -3.534 & & & & & -0.054 \\
\hline No SOC & $\mathrm{Sn}$ & 7.755 & 1.928 & -6.483 & -6.496 & & & & & -0.053 \\
\hline SOC & $\mathrm{Si}$ & 7.921 & 0.809 & -1.964 & -1.875 & -0.397 & 1.127 & -1.152 & 0.268 & -0.758 \\
\hline SOC & $\mathrm{Ge}$ & 7.903 & 1.252 & -3.481 & -3.650 & -0.489 & 1.083 & -1.118 & 0.244 & -0.247 \\
\hline SOC & $\mathrm{Sn}$ & 7.746 & 2.055 & -6.281 & -6.310 & -0.696 & 1.009 & -1.057 & 0.022 & -0.610 \\
\hline No SOC ${ }^{a}$ & $\mathrm{Ge}$ & 7.979 & 1.602 & -4.591 & -4.422 & & & & & 0.008 \\
\hline $\mathrm{SOC}^{\mathrm{a}}$ & $\mathrm{Ge}$ & 8.026 & 1.417 & -4.566 & -4.559 & -0.467 & 1.148 & -1.184 & 0.158 & 0.004 \\
\hline
\end{tabular}

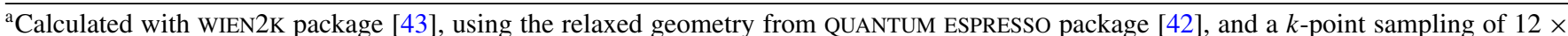
$12 \times 1$. The cutoff is $R K_{\max }=4.0$ and the muffin-tin radii are $R_{\mathrm{Te}}=2.5, R_{\mathrm{Ge}}=2.25, R_{\mathrm{Cr}}=2.5, R_{\mathrm{C}}=1.36, R_{\mathrm{W}}=2.48$, and $R_{\mathrm{S}}=2.03$. The vdW corrections and a Hubbard $U=1.0 \mathrm{eV}$ are also included.

The pattern of the in-plane contribution and the corresponding spin-momentum locking is characteristic of the Rashba SOCinduced spin texture [63].

\section{APPENDIX C: BAND STRUCTURE OF $\mathrm{Cr}_{2} \mathrm{Ge}_{2} \mathrm{Te}_{6} /$ GRAPHENE BILAYERS}

The supercell of $\mathrm{Cr}_{2} \mathrm{Ge}_{2} \mathrm{Te}_{6} /$ graphene bilayer is depicted in Fig. 8(d), where a $5 \times 5$ supercell of graphene is placed on a $\sqrt{3} \times \sqrt{3}$ supercell of $\mathrm{Cr}_{2} \mathrm{Ge}_{2} \mathrm{Te}_{6}$. We keep the lattice constant of graphene unchanged at $a=2.46 \AA$ and stretch the lattice constant of $\mathrm{Cr}_{2} \mathrm{Ge}_{2} \mathrm{Te}_{6}$ by roughly $4 \%$ from $6.8275 \AA$ [28] to $7.1014 \AA$. The resulting supercell has a lattice constant of $12.3 \AA$ and contains 80 atoms in the unit cell. The average distance between graphene and $\mathrm{Cr}_{2} \mathrm{Ge}_{2} \mathrm{Te}_{6}$ is relaxed to $3.516 \AA$, consistent with previous calculations [51]. In the case of $\mathrm{Cr}_{2} \mathrm{Si}_{2} \mathrm{Te}_{6} /$ graphene and $\mathrm{Cr}_{2} \mathrm{Sn}_{2} \mathrm{Te}_{6} /$ graphene, we get interlayer distances of $3.568 \AA$ and $3.541 \AA$, respectively.

It has been shown previously [51] that proximityinduced exchange interaction in graphene due to monolayer $\mathrm{Cr}_{2} \mathrm{Ge}_{2} \mathrm{Te}_{6}$ is an order of magnitude larger than

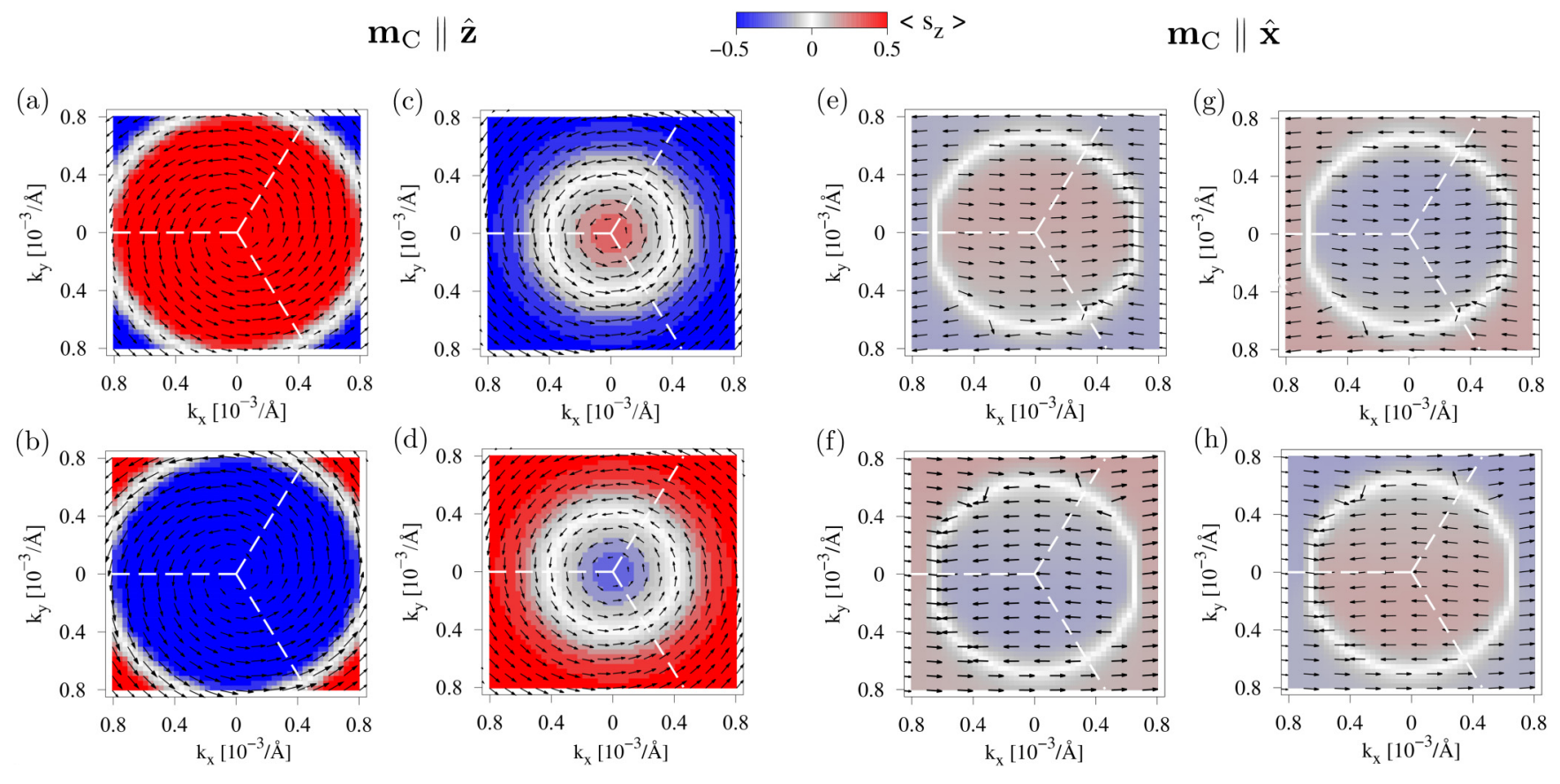

FIG. 7. The DFT-calculated equilibrium spin texture of (a) conduction and (b) valence band of $\mathrm{Cr}_{2} \mathrm{Ge}_{2} \mathrm{Te}_{6} /$ graphene/WS 2 trilayer around the K point. Color corresponds to $\left\langle\hat{s}_{z}\right\rangle$ expectation value, while arrows represent $\left\langle\hat{s}_{x}\right\rangle$ and $\left\langle\hat{s}_{y}\right\rangle$ expectation values. The dashed white lines mark the edges of the Brillouin zone. Panels (c) and (d) plot the same information as panels (a) and (b), respectively, but around the $\mathrm{K}^{\prime}$ point. Panels (e)-(h) plot the same information as panels (a)-(d), where the proximity magnetization of graphene $\mathbf{m}_{\mathrm{C}} \| \hat{\mathbf{z}}$ is orthogonal to the plane in (a)-(d) and parallel to the plane $\mathbf{m}_{\mathrm{C}} \| \hat{\mathbf{x}}$ (i.e., along the $x$ axis in Fig. 1) in panels (e)-(h). 
TABLE III. The fitting parameters of continuous Hamiltonian in Eq. (1) for $\mathrm{Cr}_{2} \mathrm{X}_{2} \mathrm{Te}_{6} /$ graphene bilayers with $\mathrm{X}=\{\mathrm{Si}$, Ge, Te $\}$. These parameters have the same meaning as those in Table II. The values of parameters fit the DFT-computed bands both with and without SOC.

\begin{tabular}{|c|c|c|c|c|c|c|c|c|c|c|}
\hline Calc. & $X$ & $\begin{array}{c}v_{\mathrm{F}} \\
{\left[10^{5} \frac{\mathrm{m}}{\mathrm{s}}\right]}\end{array}$ & $\begin{array}{c}\Delta \\
{[\mathrm{meV}]}\end{array}$ & $\begin{array}{c}\lambda_{\mathrm{ex}}^{\mathrm{A}} \\
{[\mathrm{meV}]}\end{array}$ & $\begin{array}{c}\lambda_{\mathrm{ex}}^{\mathrm{B}} \\
{[\mathrm{meV}]}\end{array}$ & $\begin{array}{c}\lambda_{\mathrm{R}} \\
{[\mathrm{meV}]}\end{array}$ & $\begin{array}{c}\lambda_{\mathrm{I}}^{\mathrm{A}} \\
{[\mathrm{meV}]}\end{array}$ & $\begin{array}{c}\lambda_{\mathrm{I}}^{\mathrm{B}} \\
{[\mathrm{meV}]}\end{array}$ & $\begin{array}{c}\xi \\
{[\mathrm{meV}]}\end{array}$ & $\begin{array}{c}E_{\mathrm{D}} \\
{[\mathrm{meV}]}\end{array}$ \\
\hline No SOC & $\mathrm{Si}$ & 8.214 & 0.005 & -1.178 & -1.243 & & & & & 29.424 \\
\hline No SOC & $\mathrm{Ge}$ & 8.175 & 0.115 & -4.705 & -4.543 & & & & & -0.269 \\
\hline No SOC & $\mathrm{Sn}$ & 8.066 & 0.119 & -6.995 & -6.894 & & & & & 0.398 \\
\hline SOC & $\mathrm{Si}$ & 8.219 & 0.062 & -1.195 & -1.166 & -0.185 & 0.122 & -0.092 & 0.204 & 10.595 \\
\hline SOC & $\mathrm{Ge}$ & 8.176 & 0.109 & -4.493 & -4.318 & -0.254 & 0.133 & -0.093 & 0.137 & 0.014 \\
\hline SOC & $\mathrm{Sn}$ & 8.069 & 0.170 & -6.830 & -6.722 & -0.480 & -0.105 & 0.118 & -0.124 & 0.760 \\
\hline No SOC ${ }^{a}$ & $\mathrm{Ge}$ & 8.204 & 0.118 & -5.986 & -5.850 & & & & & 0.001 \\
\hline $\mathrm{SOC}^{\mathrm{a}}$ & $\mathrm{Ge}$ & 8.213 & 0.114 & -5.338 & -5.216 & -0.318 & 0.149 & -0.121 & 0.037 & 0.005 \\
\hline
\end{tabular}

${ }^{a}$ Calculated with WIEN2K package [43], using a $k$-point sampling of $12 \times 12 \times 1$. The cutoff is $R K_{\max }=4.3$ and the muffin-tin radii are $R_{\mathrm{Te}}=2.5, R_{\mathrm{Ge}}=2.25, R_{\mathrm{Cr}}=2.5$, and $R_{\mathrm{C}}=1.33$. The vdW corrections and a Hubbard $U=1 \mathrm{eV}$ are also included.

proximity-induced SOC by the same monolayer. Therefore, we first perform calculations with SOC turned off. Figure 8(a) shows DFT-calculated band structure for $\mathrm{Cr}_{2} \mathrm{Ge}_{2} \mathrm{Te}_{6}$ /graphene bilayer without SOC. In general, the band structure resembles that of an isolated ferromagnetic semiconductor $\mathrm{Cr}_{2} \mathrm{Ge}_{2} \mathrm{Te}_{6}$ [27], with the graphene Dirac cone located in the gap of monolayer $\mathrm{Cr}_{2} \mathrm{Ge}_{2} \mathrm{Te}_{6}$. The linear dispersion of graphene is nicely preserved; however, the bands are spin-split due to proximity-induced exchange interaction.
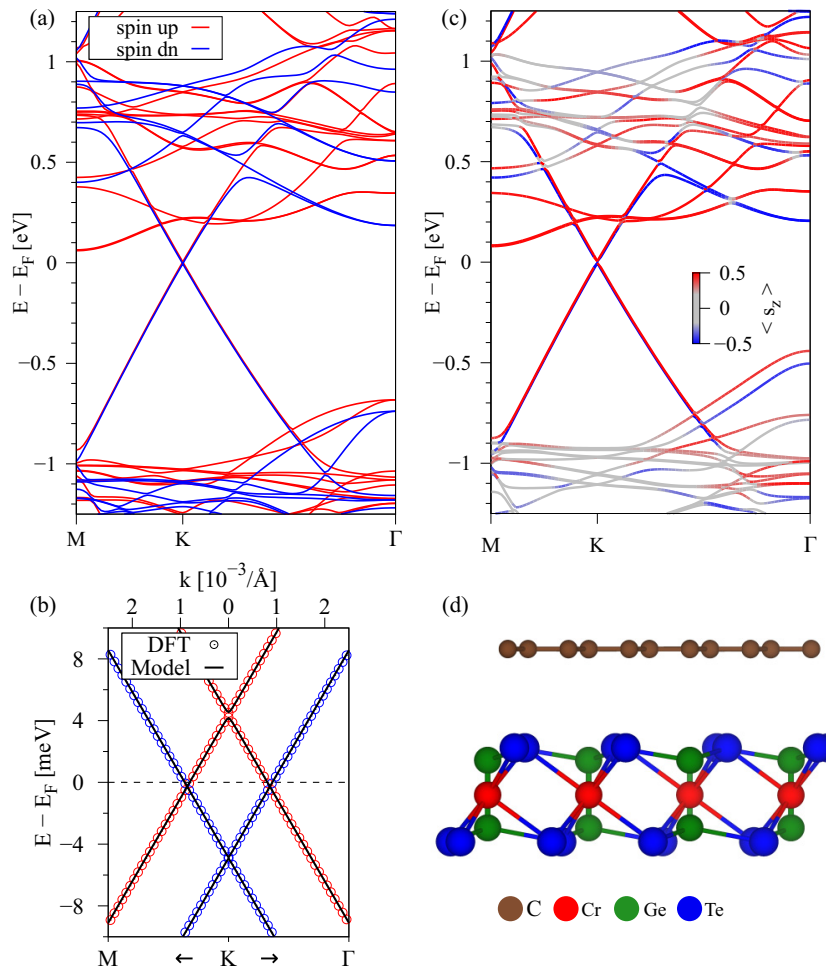

(d)

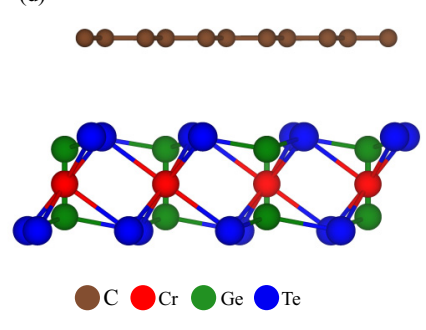

FIG. 8. (a) The DFT-calculated electronic band structure of $\mathrm{Cr}_{2} \mathrm{Ge}_{2} \mathrm{Te}_{6}$ /graphene bilayer without SOC. Bands with spin $\uparrow(\downarrow)$ are shown in red (blue). (b) A zoom to DFT-calculated low energy bands (symbols) in panel (a) at the $\mathrm{K}$ point with a fit to the continuous Hamiltonian (solid lines) in Eq. (1). (c) DFT-calculated electronic band structure of $\mathrm{Cr}_{2} \mathrm{Ge}_{2} \mathrm{Te}_{6}$ /graphene bilayer with SOC. The color corresponds to $\left\langle\hat{s}_{z}\right\rangle$ expectation value. (d) Side view of the geometry of monolayer graphene on monolayer $\mathrm{Cr}_{2} \mathrm{Ge}_{2} \mathrm{Te}_{6}$.
Without SOC, the band structure around the two valleys $\mathrm{K}$ and $\mathrm{K}^{\prime}$ are the same. When SOC is turned off, the global band gap at $E-E_{F}=0$ is absent. Figure 8(c) shows how the band structure gets modified when SOC is turned on. As demonstrated in Fig. 8(b), the bands of the continuous Hamiltonian in Eq. (1), with parameters provided in Table III, fit perfectly the DFT-calculated low energy bands in a small energy window around the Fermi level.

In addition, Fig. 9 shows an enlargement of the fine structure around the $\mathrm{K}$ and $\mathrm{K}^{\prime}$ points, including a fit with bands of the continuous Hamiltonian in Eq. (1) in the main text, when SOC is turned on. The fit can perfectly reproduce the DFT-computed band structure around both valleys. The fitting
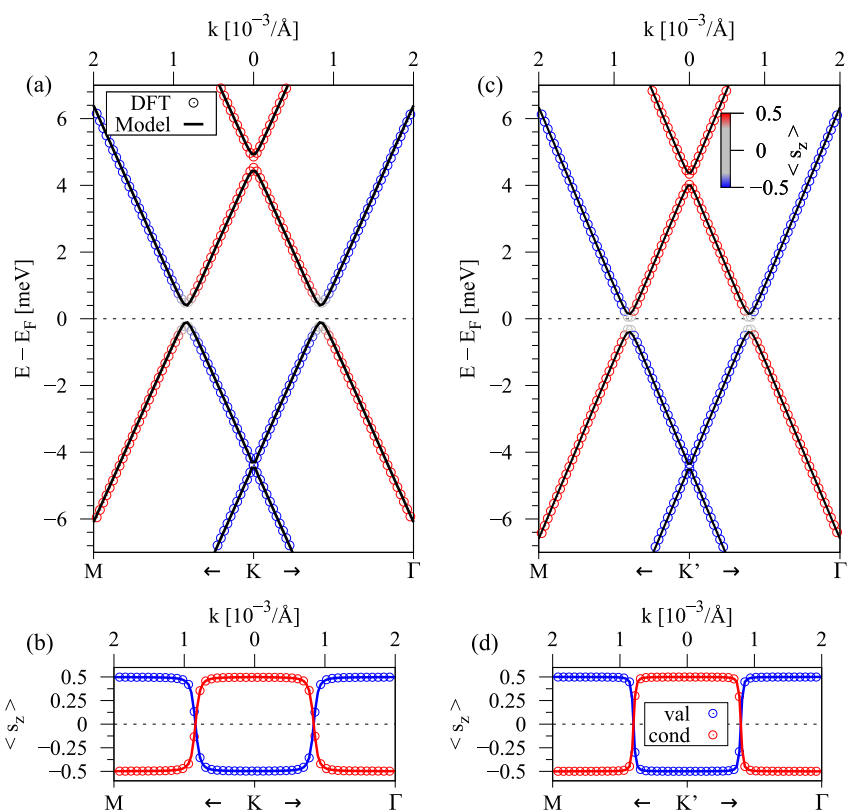

FIG. 9. (a) Zoom to DFT-calculated low energy bands in Fig. 8(c) for $\mathrm{Cr}_{2} \mathrm{Ge}_{2} \mathrm{Te}_{6} /$ graphene bilayer around the $\mathrm{K}$ point with SOC. Color of the bands corresponds to $\left\langle\hat{s}_{z}\right\rangle$ expectation value. (b) The $\left\langle\hat{s}_{z}\right\rangle$ expectation value of the valence (conduction) band is shown in blue (red). Symbols are DFT-bands and solid lines are continuous Hamiltonian [Eq. (1)] bands. Panels (c) and (d) plot the same information as panels (a) and (b), respectively, but around the $\mathrm{K}^{\prime}$ point. 
parameters are summarized in Table III for the calculations with and without SOC. We find that a tiny gap opens due to SOC, which is responsible for mixing the two spin components. The proximity exchange parameters are on the order of $5 \mathrm{meV}$, and proximity SOC is an order of magnitude smaller. As shown in Table III, DFT calculations with and without SOC show no significant change of the proximity exchange parameters $\lambda_{\mathrm{ex}}^{\mathrm{A}}$ and $\lambda_{\mathrm{ex}}^{\mathrm{B}}$, the staggered potential-induced gap $\Delta$ and the Fermi velocity $v_{\mathrm{F}}$.

In addition to matching the bands, Figs. 9(b) and 9(d) demonstrate that $\left\langle\hat{s}_{z}\right\rangle$ expectation values of the valence and conduction band agree perfectly with those from the model Hamiltonian. The bands are fully $s_{z}$ polarized due to the strong proximity exchange coupling induced by monolayer $\mathrm{Cr}_{2} \mathrm{Ge}_{2} \mathrm{Te}_{6}$. Where SOC mixes the spin- $\uparrow$ and spin- $\downarrow$ bands, a gap is opened and $\left\langle\hat{s}_{z}\right\rangle$ expectation values change in sign. The $\left\langle\hat{s}_{x}\right\rangle$ and $\left\langle\hat{s}_{y}\right\rangle$ expectation values (not shown) are very small and show a signature of Rashba SOC in agreement with the small $\lambda_{\mathrm{R}}$ parameter in Table III.

As a further consistency check, we recalculated the band structure with WIEN2K package [43]. The corresponding fitting parameters are also given in Table III. The only difference is that the proximity exchange parameters $\lambda_{\mathrm{ex}}^{\mathrm{A}}$ and $\lambda_{\mathrm{ex}}^{\mathrm{B}}$ are increased compared to the calculation with QUANTUM ESPRESSO package [42], which we can attribute to the different basis sets, number of $k$ points, and cutoffs used.

\section{APPENDIX D: TIGHT-BINDING VERSION OF CONTINUOUS GRAPHENE HAMILTONIAN}

The first-principles TB Hamiltonian employed in quantum transport calculations in Figs. 4, 5, and 6 is a discretized version of the continuous Hamiltonian in Eq. (1). Its terms are given by

$$
\begin{aligned}
\hat{H} & =\hat{H}_{0}+\hat{H}_{\Delta}+\hat{H}_{\mathrm{I}}+\hat{H}_{\mathrm{R}}+\hat{H}_{\mathrm{ex}}, \\
\hat{H}_{0} & =-t \sum_{\langle i, j\rangle} \sum_{\sigma} \hat{c}_{i \sigma}^{\dagger} \hat{c}_{j \sigma}+U_{\mathrm{tg}} \sum_{i} \sum_{\sigma} \hat{c}_{i \sigma}^{\dagger} \hat{c}_{i \sigma}, \\
\hat{H}_{\Delta} & =\sum_{\mathrm{S}=\mathrm{A}, \mathrm{B}} \sum_{i \in \mathrm{S}} \sum_{\sigma} \Delta \zeta_{\mathrm{S}} \hat{c}_{i \sigma}^{\dagger} \hat{c}_{i \sigma},
\end{aligned}
$$

$$
\begin{aligned}
\hat{H}_{\mathrm{I}} & =\sum_{\mathrm{S}=\mathrm{A}, \mathrm{B}} \frac{i \lambda_{\mathrm{I}}^{\mathrm{S}}}{3 \sqrt{3}} \sum_{\langle\langle i, j\rangle\rangle \in \mathrm{S}} \sum_{\sigma} v_{i j}\left[\hat{s}_{z}\right]_{\sigma \sigma} \hat{c}_{i \sigma}^{\dagger} \hat{c}_{j \sigma}, \\
\hat{H}_{\mathrm{R}} & =\frac{2 i \lambda_{\mathrm{R}}}{3} \sum_{\langle i, j\rangle} \sum_{\sigma \neq \sigma^{\prime}}\left[\hat{\mathbf{s}} \times \boldsymbol{d}_{i j}\right]_{\sigma \sigma^{\prime}} \hat{c}_{i \sigma}^{\dagger} \hat{c}_{j \sigma^{\prime}}, \\
\hat{H}_{\mathrm{ex}} & =\sum_{\mathrm{S}=\mathrm{A}, \mathrm{B}}\left(-\zeta_{\mathrm{S}}\right) \lambda_{\mathrm{ex}}^{\mathrm{S}} \sum_{i \in \mathrm{S}} \sum_{\sigma}\left[\mathbf{m}_{\mathrm{C}} \cdot \hat{\mathbf{s}}\right]_{\sigma \sigma} \hat{c}_{i \sigma}^{\dagger} \hat{c}_{i \sigma} .
\end{aligned}
$$

Here $\hat{c}_{i \sigma}^{\dagger}\left(\hat{c}_{i \sigma}\right)$ creates (annihilates) electron on site $i$ with spin $\sigma=\uparrow, \downarrow ; t=2.7 \mathrm{eV}$ is the nearest-neighbor hopping; $\zeta_{\mathrm{A}}=1$ for triangular sublattice $\mathrm{A}$ and $\zeta_{\mathrm{B}}=-1$ for triangular sublattice $\mathrm{B}$; sum $\langle i, j\rangle$ goes over all pairs of nearest-neighbor sites, while sum $\langle\langle i, j\rangle\rangle$ goes over all pairs of next-nearest-neighbor sites on sublattices $\mathrm{A}$ and $\mathrm{B}$; and $\mathbf{d}_{i j}$ is the vector connecting nearest neighbor sites $i$ and $j$. The parameter $v_{i j}$ is either +1 or -1 depending on the hopping direction. Other symbols have the same meaning as in Eq. (1), and the values of the fitting parameters are the same as in Table II.

The only difference between the continuous Hamiltonian in Eq. (1) and the TB Hamiltonian in Eq. (D1) is neglect of $\hat{H}_{\xi}$ term in Eq. (D1) due to small value of $\xi$ parameter in Table II. Another difference is that $\mathbf{m}_{\mathrm{C}}$ as the unit vector along the proximity-induced magnetization of graphene in $\hat{H}_{\mathrm{ex}}$ can point in any direction to make it possible to study the angular dependence of the SO torque in Fig. 6. In contrast, this term is fixed only along the $z$ axis in Eq. (1).

We note that quantum transport calculations using TB Hamiltonian in Eq. (D1) are equivalent to computationally much more expensive calculations using DFT Hamiltonian [8], as long as one remains in the linear-response transport regime driven by small applied bias voltage. The small bias voltage $V_{b} \ll E_{F}$ is indeed utilized in the calculations in Figs. 4, 5, and 6. This is due to the fact that self-consistent recalculation of particle densities and the Hamiltonian due to current flow is nonlinear effect in the bias voltage. Trivially, one should also keep the Fermi energy $E_{F}$ in Figs. 4 and 6 within the energy window around the Dirac point where the continuous Hamiltonians in Eq. (1) or TB Hamiltonian in Eq. (D1) with parameters in Table II are applicable.
[1] A. Manchon, I. M. Miron, T. Jungwirth, J. Sinova, J. Zelezný, A. Thiaville, K. Garello, and P. Gambardella, Current-induced spin-orbit torques inferromagnetic and antiferromagnetic systems, Rev. Mod. Phys. 91, 035004 (2019).

[2] N. Locatelli, V. Cros, and J. Grollier, Spin-torque building blocks, Nat. Mater. 13, 11 (2014).

[3] R. Ramaswamy, J. M. Lee, K. Cai, and H. Yang, Recent advances in spin-orbit torques: Moving towards device applications, Appl. Phys. Rev. 5, 031107 (2018).

[4] W. A. Borders, H. Akima, S. Fukami, S. Moriya, S. Kurihara, Y. Horio, S. Sato, and H. Ohno, Analogue spinorbit torque device for artificial-neural-network-based associative memory operation, Appl. Phys. Expr. 10, 013007 (2017).

[5] Y. Wang, D. Zhu, Y. Wu, Y. Yang, J. Yu, R. Ramaswamy, R. Mishra, S. Shi, M. Elyasi, K.-L. Teo, Y. Wu, and H. Yang,
Room temperature magnetization switching in topological insulator-ferromagnet heterostructures by spin-orbit torques, Nat. Commun. 8, 1364 (2017).

[6] S. Shi et al., All-electric magnetization switching and Dzyaloshinskii-Moriya interaction in $\mathrm{WTe}_{2}$ /ferromagnet heterostructures, Nat. Nanotech. 14, 945 (2019).

[7] L. Zhu, D. C. Ralph, and R. A. Buhrman, Spin-Orbit Torques in Heavy-Metal-Ferromagnet Bilayers with Varying Strengths of Interfacial Spin-Orbit Coupling, Phys. Rev. Lett. 122, 077201 (2019).

[8] K. Dolui, M. D. Petrović, K. Zollner, P. Plecháč, J. Fabian, and B. K. Nikolić, Proximity spin-orbit torque on a two-dimensional magnet within van der Waals heterostructure: Current-driven antiferromagnet-to-ferromagnet reversible nonequilibrium phase transition in bilayer $\mathrm{CrI}_{3}, \mathrm{Nano}$ Lett. 20 , 2288 (2020). 
[9] M. Gibertini, M. Koperski, A. F. Morpurgo, and K. S. Novoselov, Magnetic 2D materials and heterostructures, Nat. Nanotech. 14, 408 (2019).

[10] D. L. Cortie, G. L. Causer, K. C. Rule, H. Fritzsche, W. Kreuzpaintner, and F. Klose, Two-dimensional magnets: Forgotten history and recent progress towards spintronic applications, Adv. Funct. Mater. 30, 1901414 (2020).

[11] W. Lv, Z. Jia, B. Wang, Y. Lu, X. Luo, B. Zhang, Z. Zeng, and Z. Liu, Electric-field control of spin-orbit torques in $\mathrm{WS}_{2}$ /permalloy bilayers, ACS Appl. Mater. Interfaces 10, 2843 (2018).

[12] S. Yan and Y. B. Bazaliy, Phase diagram and optimal switching induced by spin Hall effect in a perpendicular magnetic layer, Phys. Rev. B 91, 214424 (2015).

[13] J. Yoon, S.-W. Lee, J. H. Kwon, J. M. Lee, J. Son, X. Qiu, K.-J. Lee, and H. Yang, Anomalous spin-orbit torque switching due to field-like torque-assisted domain wall reflection, Sci. Adv. 3, e1603099 (2017).

[14] J. Sinova, S. O. Valenzuela, J. Wunderlich, C. H. Back, and T. Jungwirth, Spin Hall effects, Rev. Mod. Phys. 87, 1213 (2015).

[15] K. Dolui and B. K. Nikolić, Spin-memory loss due to spin-orbit coupling at ferromagnet/heavy-metal interfaces: Ab initio spindensity matrix approach, Phys. Rev. B 96, 220403(R) (2017).

[16] K. D. Belashchenko, A. A. Kovalev, and M. van Schilfgaarde, Theory of Spin Loss at Metallic Interfaces, Phys. Rev. Lett. 117, 207204 (2016).

[17] K. Gupta, R. J. H. Wesselink, R. Liu, Z. Yuan, and P. J. Kelly, Disorder Dependence of Interface Spin Memory Loss, Phys. Rev. Lett. 124, 087702 (2020)

[18] K. D. Belashchenko, A. A. Kovalev, and M. van Schilfgaarde, Interfacial contributions to spin-orbit torque and magnetoresistance in ferromagnet/heavy-metal bilayers, Phys. Rev. B 101, 020407 (2020).

[19] F. Mahfouzi, R. Mishra, P.-H. Chang, H. Yang, and N. Kioussis, Microscopic origin of spin-orbit torque in ferromagnetic heterostructures: A first-principles approach, Phys. Rev. B 101, 060405 (2020).

[20] K.-W. Kim, K.-J. Lee, J. Sinova, H.-W. Lee, and M. D. Stiles, Spin-orbit torques from interfacial spin-orbit coupling for various interfaces, Phys. Rev. B 96, 104438 (2017).

[21] V. P. Amin, J. Zemen, and M. D. Stiles, Interface-Generated Spin Currents, Phys. Rev. Lett. 121, 136805 (2018).

[22] S. Ghosh and A. Manchon, Spin-orbit torque in a threedimensional topological insulator-ferromagnet heterostructure: Crossover between bulk and surface transport, Phys. Rev. B 97, 134402 (2018).

[23] D. A. Pesin and A. H. MacDonald, Quantum kinetic theory of current-induced torques in Rashba ferromagnets, Phys. Rev. B 86, 014416 (2012).

[24] A. Qaiumzadeh, R. A. Duine, and M. Titov, Spin-orbit torques in two-dimensional Rashba ferromagnets, Phys. Rev. B 92, 014402 (2015).

[25] I. A. Ado, O. A. Tretiakov, and M. Titov, Microscopic theory of spin-orbit torques in two dimensions, Phys. Rev. B 95, 094401 (2017).

[26] H. Kurebayashi et al., An antidamping spin-orbit torque originating from the Berry curvature, Nat. Nanotechnol. 9, 211 (2014).

[27] G. Menichetti, M. Calandra, and M. Polini, Electronic structure and magnetic properties of few-layer $\mathrm{Cr}_{2} \mathrm{Ge}_{2} \mathrm{Te}_{6}$ : The key role of nonlocal electron-electron interaction effects, 2D Mater. 6, 045042 (2019).

[28] V. Carteaux, D. Brunet, G. Ouvrard, and G. Andre, Crystallographic, magnetic and electronic structures of a new layered ferromagnetic compound $\mathrm{Cr}_{2} \mathrm{Ge}_{2} \mathrm{Te}_{6}$, J. Phys.: Condens. Mat. 7, 69 (1995).

[29] M. Gmitra, D. Kochan, P. Högl, and J. Fabian, Trivial and inverted Dirac bands and the emergence of quantum spin Hall states in graphene on transition-metal dichalcogenides, Phys. Rev. B 93, 155104 (2016).

[30] M. Gmitra, S. Konschuh, C. Ertler, C. Ambrosch-Draxl, and J. Fabian, Band-structure topologies of graphene: Spin-orbit coupling effects from first principles, Phys. Rev. B 80, 235431 (2009).

[31] F. J. Sousa, G. Tatara, and A. Ferreira, Emergent spin-orbit torques in two-dimensional material/ferromagnet interfaces, arXiv:2005.09670.

[32] F. Freimuth, S. Blügel, and Y. Mokrousov, Spin-orbit torques in $\mathrm{Co} / \mathrm{Pt}(111)$ and $\mathrm{Mn} / \mathrm{W}(001)$ magnetic bilayers from first principles, Phys. Rev. B 90, 174423 (2014).

[33] B. K. Nikolić, K. Dolui, M. Petrović, P. Plecháč, T. Markussen, and K. Stokbro, First-principles quantum transport modeling of spin-transfer and spin-orbit torques in magnetic multilayers, in Handbook of Materials Modeling: Applications: Current and Emerging Materials, edited by W. Andreoni and S. Yip (Springer, Cham, 2018).

[34] K. Dolui and B. K. Nikolić, Spin-orbit-proximitized ferromagnetic metal by monolayer transition metal dichalcogenide: Atlas of spectral functions, spin textures and spin-orbit torques in $\mathrm{Co} / \mathrm{MoSe}_{2}, \mathrm{Co} / \mathrm{WSe}_{2}$ and $\mathrm{Co} / \mathrm{TaSe}_{2}$ heterostructures, arXiv:2006.11335.

[35] K. D. Belashchenko, A. A. Kovalev, and M. van Schilfgaarde, First-principles calculation of spin-orbit torque in a Co/Pt bilayer, Phys. Rev. Mater. 3, 011401 (2019).

[36] G. Stefanucci and R. van Leeuwen, Nonequilibrium Many-Body Theory of Quantum Systems: A Modern Introduction (Cambridge University Press, Cambridge, UK, 2013).

[37] P.-H. Chang, T. Markussen, S. Smidstrup, K. Stokbro, and B. K. Nikolić, Nonequilibrium spin texture within a thin layer below the surface of current-carrying topological insulator $\mathrm{Bi}_{2} \mathrm{Se}_{3}$ : A first-principles quantum transport study, Phys. Rev. B 92, 201406(R) (2015).

[38] A. Kalitsov, S. A. Nikolaev, J. Velev, M. Chshiev, and O. Mryasov, Intrinsic spin-orbit torque in a single-domain nanomagnet, Phys. Rev. B 96, 214430 (2017).

[39] A. F. Young and P. Kim, Quantum interference and Klein tunnelling in graphene heterojunctions, Nat. Phys. 5, 222 (2009).

[40] M.-H. Liu and K. Richter, Efficient quantum transport simulation for bulk graphene heterojunctions, Phys. Rev. B 86, 115455 (2012).

[41] M. Milletarì, M. Offidani, A. Ferreira, and R. Raimondi, Covariant Conservation Laws and the Spin Hall Effect in DiracRashba Systems, Phys. Rev. Lett. 119, 246801 (2017).

[42] P. Giannozzi et al., QUANTUM ESPRESSO: A modular and opensource software project for quantum simulations of materials, J. Phys.: Condens. Mat. 21, 395502 (2009).

[43] P. Blaha, K. Schwarz, G. K. H. Madsen, D. Kvasnicka, J. Luitz, R. Laskowski, F. Tran, and L. D. Marks, WIEN2k: An Augmented Plane Wave Plus Local Orbitals Program for 
Calculating Crystal Properties (Vienna University of Technology, Vienna, 2019).

[44] V. T. Phong, N. R. Walet, and F. Guinea, Effective interactions in a graphene layer induced by the proximity to a ferromagnet, 2D Mater. 5, 014004 (2017).

[45] D. Kochan, S. Irmer, and J. Fabian, Model spin-orbit coupling Hamiltonians for graphene systems, Phys. Rev. B 95, 165415 (2017).

[46] J. M. Marmolejo-Tejada, P.-H. Chang, P. Lazić, S. Smidstrup, D. Stradi, K. Stokbro, and B. K. Nikolić, Proximity band structure and spin textures on both sides of topologicalinsulator/ferromagnetic-metal interface and their charge transport probes, Nano Lett. 17, 5626 (2017).

[47] I. Žutić, A. Matos-Abiague, B. Scharf, H. Dery, and K. Belashchenko, Proximitized materials, Mater. Today 22, 85 (2019).

[48] M. Gmitra and J. Fabian, Graphene on transition-metal dichalcogenides: A platform for proximity spin-orbit physics and optospintronics, Phys. Rev. B 92, 155403 (2015).

[49] A. Hallal, F. Ibrahim, H. Yang, S. Roche, and M. Chshiev, Tailoring magnetic insulator proximity effects in graphene: First-principles calculations, 2D Mater. 4, 025074 (2017).

[50] B. Karpiak, A. W. Cummings, K. Zollner, M. Vila, D. Khokhriakov, A. M. Hoque, A. Dankert, P. Svedlindh, J. Fabian, and S. Roche, Magnetic proximity in a van der Waals heterostructure of magnetic insulator and graphene, 2D Mater. 7, 015026 (2019).

[51] J. Zhang, B. Zhao, Y. Yao, and Z. Yang, Robust quantum anomalous Hall effect in graphene-based van der Waals heterostructures, Phys. Rev. B 92, 165418 (2015).

[52] K. Garello, I. M. Miron, C. O. Avci, F. Freimuth, Y. Mokrousov, S. Blügel, S. Auffret, O. Boulle, G. Gaudin, and P. Gambardella, Symmetry and magnitude of spin-orbit torques in ferromagnetic heterostructures, Nat. Nanotech. 8, 587 (2013).
[53] V. Edelstein, Spin polarization of conduction electrons induced by electric current in two-dimensional asymmetric electron system, Solid State Commun. 73, 233 (1990).

[54] A. G. Aronov and Yu. B. Lyanda-Geller, Nuclear electric resonance and orientation of carrier spins by an electric field, JETP Lett. 50, 431 (1989).

[55] M. Offidani, M. Milletarí, R. Raimondi, and A. Ferreira, Optimal Charge-to-Spin Conversion in Graphene on TransitionMetal Dichalcogenides, Phys. Rev. Lett. 119, 196801 (2017).

[56] C. H. Lewenkopf, E. R. Mucciolo, and A. H. Castro Neto, Numerical studies of conductivity and Fano factor in disordered graphene, Phys. Rev. B 77, 081410(R) (2008).

[57] H. Kohno, G. Tatara, and J. Shibata, Microscopic calculation of spin torques in disordered ferromagnets, J. Phys. Soc. Japan 75, 113706 (2006).

[58] W. J. Schutte, J. L. De Boer, and F. Jellinek, Crystal structures of tungsten disulfide and diselenide, J. Solid State Chem. 70, 207 (1987).

[59] J. P. Perdew, K. Burke, and M. Ernzerhof, Generalized Gradient Approximation Made Simple, Phys. Rev. Lett. 77, 3865 (1996).

[60] G. Kresse and D. Joubert, From ultrasoft pseudopotentials to the projector augmented-wave method, Phys. Rev. B 59, 1758 (1999).

[61] S. Grimme, Semiempirical GGA-type density functional constructed with a long-range dispersion correction, J. Comput. Chem. 27, 1787 (2006).

[62] V. Barone, M. Casarin, D. Forrer, M. Pavone, M. Sambi, and A. Vittadini, Role and effective treatment of dispersive forces in materials: Polyethylene and graphite crystals as test cases, J. Comput. Chem. 30, 934 (2009).

[63] A. Manchon, H. C. Koo, J. Nitta, S. M. Frolov, and R. A. Duine, New perspectives for Rashba spin-orbit coupling, Nat. Mater. 14, 871 (2015). 